\title{
Bedside lung ultrasound score (LUSS) on assessing pneumonia in COVID-19 neonates
}

\author{
Wei $\mathrm{Li}^{1}$, Manli Fu${ }^{1}$, Chao Qian ${ }^{2}$, Xin Liu ${ }^{1}$, Lingkong Zeng ${ }^{1}$, Huan Zhou ${ }^{1}$, and Li Yuan ${ }^{1}$ \\ ${ }^{1}$ Wuhan Children' s Hospital Wuhan Maternal and Child Healthcare HospitalTongji \\ Medical CollegeHuazhong University of Science \& Technology \\ ${ }^{2}$ Wuhan Children's Hospital (Wuhan Maternal and Child Healthcare Hospital, Tongji \\ Medical College, Huazhong University of Science \& Technology
}

September 24, 2020

\begin{abstract}
We used the lung ultrasound score (LUSS) to analyze the ultrasound images and assess the lesions and aeration loss in 12 lung regions of 11 COVID-19 neonates born to mother with COVID-19 and 11 age- and gender-matched controls. In the COVID-19 group, 132 regions were reviewed and 83 regions (62.8\%) detected abnormalities. Compared with controls, COVID-19 neonates showed more increased B-lines (83 regions), abnormal A-lines (83 regions), abnormal pleural line (29 regions) and subpleural consolidation (5 regions). among which 49 regions (37\%) were normal, 73 regions (55\%) scored 1, and 10 regions (8\%) scored 2. The LUSS was significantly higher in COVID-19 group. All the lesions were bilateral, multiple regions involved, and mainly located in bilateral lower lobes and right middle lobe. The intra-observer and inter-observer reproducibility of LUSS were excellent. Lung ultrasound is a noninvasive and convenient method for the assessment of neonatal COVID-19 pneumonia and presents typical signs. LUSS provides valuable semi-quantitative information about the lesion distribution and severity.
\end{abstract}

\section{Introduction}

The 2019 novel coronavirus, or severe acute respiratory syndrome coronavirus-2 (SARS-CoV-2) that results in coronavirus disease 2019 (COVID-19), has been declared a pandemic and is severely affecting the public health worldwide. The epidemic geographically focused in the city of Wuhan, Hubei in mainland China during Jan to Mar 2020. With the sharp increase in the number of infections, the number of pregnant women and children with COVID-19 is also on the rise. The neonates born to mothers with COVID-19 were investigated in isolation ward, and some of them were identified with COVID-19 ${ }^{1}$. CT scan is the primary diagnostic method of COVID-19, with specific imaging findings ${ }^{1}$. Bedside lung ultrasound (LUS) examination is convenient and non-ionizing, and widely used in the assessment or monitoring of patients with infective pneumonia and dyspnea, especially for COVID-19 neonates. Lung ultrasound score (LUSS) ${ }^{2}$ is capable of defining severe loss of aeration, which is helpful in semi-quantitative evaluation of neonatal COVID19 pneumonia. However, the morbidity of COVID-19 in pediatric population was low. Our hospital was the only designated children hospital in Wuhan for treating COVID-19. In this single center pioneer study, we explored the ultrasonic features and severity of aeration loss of neonatal COVID-19 using bedside lung ultrasound score method.

\section{Methods}

\section{Study population and clinical information}

We retrospectively surveyed all neonates born to mothers with COVID-19 from January 31 to March 31 2020, and investigated 11 neonates with confirmed SARS-CoV-2 infection in COVID-19 group, and 11 
normal neonates in control group.

All the clinical materials and lung ultrasound images were analyzed in both groups. This study was approved by the local medical ethics committee. Written informed consent was obtained from the neonates' parents. The diagnosis and management of neonate with or at risk of COVID-19 were in accordance with guidelines provided by the National Health Commission and the Chinese Perinatal-Neonatal SARS-CoV-2 Committee ${ }^{3}$.

Data were collected from. regarding demographic, epidemiologic, and clinical features were obtained from the medical records system. In addition, SARS-CoV-2 real- time RT-PCR tests (Novel Coronavirus PCR Fluorescence Diagnostic Kit, Da An Gene, Guangdong, P. R. China) were conducted using nasopharyngeal swab samples. SARS-CoV-2 specific serum IgM and IgG test were performed. All the neonates underwent influenzas virus screening and tested negatives.

The inclusive criteria were: neonates born to mothers with COVID-19; positive results in SARS-CoV-2 real-time RT-PCR tests using nasopharyngeal swabs or positive results in SARS-CoV-2 specific IgM using serum. The exclusive criteria were other etiological neonatal pneumonia, combined with other congenital abnormalities.

\section{Lung ultrasound (LUS) and Lung ultrasound Scores (LUSS)}

Bedside LUS exams were acquired during the neonatal hospitalization by one doctor over 5-year experience on LUS. Pacifier or sedation could be used in some neonates if uncooperative. Images were collected by a portable ultrasound system (Mindray M9) with a high-frequency (L12-4S,4-12MHz), linear-array probe. The neonates were placed in a supine, prone or side position during the exams.

We divided each lung into 6 regions and labelled them, by using the anterior axillary line, posterior axillary line and the line connecting the nipples (Fig. 1). The most pathological pattern acquired in each intercostal space during an entire respiratory cycle was retained for the further analysis and considered representative of the area score. The longitudinal scan was done first, to correctly identify the pleura; a clip at least as long as one respiratory cycle was stored for offline analysis. The transversal scan was obtained by a rotation centered on the pleura, until complete disappearance of the ribs; a second clip was stored. Both longitudinal and transversal sections were collected on the anterior, lateral, and posterior chest wall.

e utilized semi-quantitative LUSS, grading between 0 and 3, depending on the severity of aeration loss in each lung region (Table 1 ). The following images were recorded ${ }^{2}$ and scored: presence of A-lines, maximum number of B-lines, visual percentage of lung area occupied by confluent B-lines, visual pleural involvement $>50 \%$ or [?]50\%, and tissue-like patterns (consolidations).

LUSS was independently assessed by two experienced ultrasonic doctors for inter-observer reproducibility. Intra-observer analysis was performed by using the recorded images 4 weeks after the initial reading was conducted.

\section{Statistical analysis}

Data were statistically analyzed using SPSS 23.0 and MedCalc 16.2. Data were expressed as mean \pm SD or as d number (percentage). Difference of variance was calculated by a variance ratio test (F-test). To compare continuous variables, we used the independent sample t test. For categorical variables, we applied the Chi-square test.

The Inter- and intra-observer reproducibility of LUSS was assessed by using Bland-Altman plot, with a coefficient of variation, Pearson's correlation, and intra-class correlation coefficient (ICC). The coefficient of variation was defined as the standard deviation of differences between two readings in the percentage of the mean. $\mathrm{P}$ values $<0.05$ were considered statistically significant.

\section{Results}

\section{Clinical information of the study population}


Eleven neonates (3 males, age 3.8+- 5.2 days) were enrolled in COVID-19 group. Demographic characteristics and clinical features of COVID-19 group and control group are described in Table 2. There were no differences in demographic characteristics and between 2 groups. In COVID-19 group, all the neonates initially experienced mild complaints, i.e. shortness of breath, increasing respiratory rate, and showed pneumonia on chest CT or chest X-ray. There were $5(45 \%)$ neonates were positive results in SARS-CoV-2 nasopharyngeal swabs tests, and 6 patients were positive results in specific IgM and IgG serum tests. The values of IgM and IgG in this group were $29.5+-27.7$ and $73.9+-31.7 \mathrm{AU} / \mathrm{ml}$, respectively.

\section{Lung ultrasound and LUSS}

In COVID-19 group, there were totally 132 regions reviewed and 83 regions (62.8\%) detected abnormalities. Each patient showed abnormalities in bilateral lungs and multiple regions, with maximal 10 regions, average 7.5 regions involved. The majority of detectable ultrasonic signs were increased B-lines (83 regions, $62.8 \%$ ), from sparse (73 regions, 55.3\%) B-lines to confluent B-lines (10 regions, 7.6\%); abnormal scored 1, 10 regions (8\%) scored 2 and no regions scored 0. Lung score was obviously higher in COVID-19 group than that in control group (Fig. 3). The lesions were primarily located in bilateral lower A-lines (83 regions, 62.8\%), from decreased A-lines (52 regions, 39.3\%) to disappear of A-line (31 regions, 23.4\%); blurring, irregularities, interruption of pleural line (29 regions, 21.9\%), subpleural consolidation ( 5 regions, $3.8 \%$ ), and no pleural effusion (Fig. 2). There were 49 regions $(37 \%)$ were normal, 73 regions $(55 \%)$ lobes and right middle lobe (region L4, L5, L6, R3, R4, R5, R6) (Table 3).

\section{Inter-and intro-operator agreement}

Bland-Alterman analysis revealed that LUSS derived by 2 readings from intra- and inter-observers, were highly consistent (Fig.4). Both the intra- and inter-observer reproducibility were excellent, with ICCs of 0.930 and 0.854 , respectively. Intra-observer reproducibility of LUSS slightly better than that of interobserver (Table 4).

\section{Discussion}

The SARS-Cov-2 particles enter the airways and lungs, and could reach the bronchioles and alveoli. Therefore, the lesions mostly located in the terminal alveoli and subpleural ${ }^{4}$. The pathologic findings ${ }^{5}$ of COVID-19 showed diffuse lesions of the alveoli with mucus exudation, which caused consolidation of the lung. Infants and children are also considered to be susceptible to COVID-19, and mainly infected from family cluster outbreak $^{6}$. Compared with infected adults, infected children experienced differently, and most appear to have a milder clinical course. Asymptomatic patients are not uncommon in pediatric population, some of whom have radiologic features of pneumonia ${ }^{7}$. Therefore, it is important for us to recognize the pediatric COVID19 patients, using imaging modalities. Our colleague has reported ${ }^{1} 3$ neonates were identified SARS-CoV-2 infection and associated pneumonia in 33 neonates born to mothers with COVID-19. Our study demonstrated the similar scene, and all the COVID-19 neonates showed mild symptoms and pneumonia on chest CT or X-ray.

CT features in pediatric patients with COVID-19 infection showed different points from adults. Most of children stay in the early stage, and less move into the advanced and critical stage. On CT images, ground glass opacities (GGO) appear near the pleura, but pleural effusion is rare. However, compared to adults, the lesions distribution is less extensive and some atypical GGO appear in children ${ }^{8}$. Cause most of pediatric COVID-19 cases stay in the early stage and located in the peripheral area under the pleura with limited extension towards the pulmonary hilum, ultrasonic modality could also show the distribution of COVID-19 lesions.

Lung ultrasound (LUS) cannot create direct imaging of the pulmonary parenchyma, but can be used for the diagnosis by utilizing artefacts formed by different pathological changes. When air-to-liquid ratio of the lungs is changed by specific lung diseases, especially when the pleura gets involved, LUS images can be changed and help located the lung lesions. All LUS signs and patterns described in the adult are alike in neonates and children, in both normal and pathological conditions ${ }^{9}$. The advantage of LUS in pediatric population is 
also related to the small size of the chest, which allows an optimal, visualization of the lungs in most cases, irrespective of the depth of the main target of the examination. A number of studies have described the benefits of LUS in the diagnosis of TTN, RDS, bronchiolitis in neonates. Compared with chest X-ray, LUS is valuable in detecting pediatric pneumonia with good sensitivity and specificity, especially companied with lung consolidation ${ }^{10}$. However, it can reduce $38.8 \%$ CXR usage $^{11}$ in pediatric population.

Considering children's higher radio-sensitivity and free radiation of this technique, neonates may benefit from LUS. Launching a LUS in their neonatal intensive care unit (NICU) roughly halved the number of chest radiograms and significantly decreased the mean radiation dose ${ }^{12}$. In fact, LUS exam during COVID-19 outbreak should be as focused as necessary to obtain diagnostic views ${ }^{12}$, but should also be comprehensive enough to avoid return to the isolation ward. Each exam should be tailored to the indication and planned in advance.

In current study, LUS presented most of the lung regions involved, and the lesions are bilateral and diffuse. Lesions distribution suggested that bilateral lower lobes and right middle lobe were mostly infected, which were similar with CT findings ${ }^{4,8}$. Abnormalities in B-lines and A-lines were the most common signs, covering all the infected regions. There may be a few B-lines in the lung fields of normal neonates at the age of 3-7 days. The abnormal increase of B-lines, usually accompanied with disappear of A-lines, represents fluid accumulation at the alveolar level and lobular space, decreased air-to-liquid ratio, and pulmonary function impairment in varying degrees. When confluent B-lines spread throughout the lung field, the sonogram shows a diffuse B-line pattern, called the white lung, which wasn't observed in our study due to mildly symptomatic pediatric patients ${ }^{13}$. LUS showed a high sensitivity and specificity to detect lung edema by abnormal B-lines, however it was challenging to identify the etiology of edema, i.e. cardiogenic, nephrogenic or pneumonia ${ }^{14}$. We propose that it could be combined with other organ clinical and ultrasonic assessment to give a comprehensive judgment of the situation. Subpleural consolidation was another typical sign in neonatal COVID-19. It indicates lung tissue becomes non-aerated and echogenic, resulting in ultrasonic solid tissue change. If residual gas or liquid in the bronchi are visible, air bronchogram or fluid bronchogram can be presented. In our study, we only observed limited subpleural consolidation, which is inconsistent with local subpleural lesions displayed in CT images.

Since LUS detects the artefacts generated by the accumulation of fluid, we could rank the artefacts according to the air-liquid ratio and create scores reflecting lung aeration. LUSS, a three-stage classification system, could comprehensively and semi-quantitatively reflect the lung aeration function and disease severity. LUSS is well correlated with indices of oxygenation in both term and preterm neonates ${ }^{15}$, guide surfactant therapy. LUSS could assess global and regional lung aeration, and well correlated with CT quantitative analysis indices ${ }^{16}$ in ARDS. In present study, the global LUSS was obviously higher in COVID-19 group, in consistent with patients' respiratory symptoms. The regional score also reflected variant lung aeration and severity of infectious lesions, especially in bilateral lower lobes and right middle lobe. LUSS calculation has a high inter-observer agreement regardless of the ultrasonographers' experience ${ }^{15}$, which has also been validated in our study.

In present study, we presented the typical ultrasonic features of neonatal COVID-19 pneumonia using bedside ultrasound machine. LUSS, with excellent intra-observer and inter-observer reproducibility, can semiquantitatively assess global and regional lung aeration and disease severity. In clinic settings, we should keep in mind that combining the imaging findings with clinic, epidemiological and etiological evidence to diagnose COVID-19. The limitation of the study was small sample size due to low morbidity in neonates. However, LUS is a free radiational, convenient, non-invasive, reproducible and reliable imaging modality in neonatal COVID-19 pneumonia diagnosis and monitoring.

\section{References}

1. Zeng L, Xia S, Yuan W, Yan K, Xiao F, Shao J, Zhou W. Neonatal Early-Onset Infection With SARSCoV-2 in 33 Neonates Born to Mothers With COVID-19 in Wuhan, China. JAMA PEDIATR 2020.

2. Mongodi S, Bouhemad B, Orlando A, Stella A, Tavazzi G, Via G, Iotti GA, Braschi A, Mojoli F. 
Modified Lung Ultrasound Score for Assessing and Monitoring Pulmonary Aeration. ULTRASCHALL MED 2017;38(5):530-537.

3. Wang L, Shi Y, Xiao T, Fu J, Feng X, Mu D, Feng Q, Hei M, Hu X, Li Z, Lu G, Tang Z, Wang Y, Wang C, Xia S, Xu J, Yang Y, Yang J, Zeng M, Zheng J, Zhou W, Zhou X, Zhou X, Du L, Lee SK, Zhou W. Chinese expert consensus on the perinatal and neonatal management for the prevention and control of the 2019 novel coronavirus infection (First edition). Ann Transl Med 2020;8(3):47.

4. Xu YH, Dong JH, An WM, Lv XY, Yin XP, Zhang JZ, Dong L, Ma X, Zhang HJ, Gao BL. Clinical and computed tomographic imaging features of novel coronavirus pneumonia caused by SARS-CoV-2. J Infect 2020;80(4):394-400.

5. Xu Z, Shi L, Wang Y, Zhang J, Huang L, Zhang C, Liu S, Zhao P, Liu H, Zhu L, Tai Y, Bai C, Gao T, Song J, Xia P, Dong J, Zhao J, Wang FS. Pathological findings of COVID-19 associated with acute respiratory distress syndrome. Lancet Respir Med 2020;8(4):420-422.

6. Cai J, Xu J, Lin D, Yang Z, Xu L, Qu Z, Zhang Y, Zhang H, Jia R, Liu P, Wang X, Ge Y, Xia A, Tian H, Chang H, Wang C, Li J, Wang J, Zeng M. A Case Series of children with 2019 novel coronavirus infection: clinical and epidemiological features. CLIN INFECT DIS 2020.

7. Lu X, Xiang Y, Du H, Wing-Kin WG. SARS-CoV-2 infection in children - Understanding the immune responses and controlling the pandemic; 2020.

8. Xia W, Shao J, Guo Y, Peng X, Li Z, Hu D. Clinical and CT features in pediatric patients with COVID-19 infection: Different points from adults. Pediatr Pulmonol 2020;55(5):1169-1174.

9. Lichtenstein DA, Mauriat P. Lung Ultrasound in the Critically Ill Neonate. Curr Pediatr Rev 2012;8(3):217-223.

10. Shah VP, Tunik MG, Tsung JW. Prospective evaluation of point-of-care ultrasonography for the diagnosis of pneumonia in children and young adults. JAMA PEDIATR 2013;167(2):119-125.

11. Jones BP, Tay ET, Elikashvili I, Sanders JE, Paul AZ, Nelson BP, Spina LA, Tsung JW. Feasibility and Safety of Substituting Lung Ultrasonography for Chest Radiography When Diagnosing Pneumonia in Children: A Randomized Controlled Trial. CHEST 2016;150(1):131-138.

12. Escourrou G, De Luca D. Lung ultrasound decreased radiation exposure in preterm infants in a neonatal intensive care unit. ACTA PAEDIATR 2016;105(5): e237-e239.

13. Raimondi F, Migliaro F, Sodano A, Umbaldo A, Romano A, Vallone G, Capasso L. Can neonatal lung ultrasound monitor fluid clearance and predict the need of respiratory support? CRIT CARE 2012;16(6): R220.

14. Dietrich CF, Mathis G, Blaivas M, Volpicelli G, Seibel A, Wastl D, Atkinson NS, Cui XW, Fan M, Yi D. Lung B-line artefacts and their use. J THORAC DIS 2016;8(6):1356-1365.

15. Brat R, Yousef N, Klifa R, Reynaud S, Shankar AS, De Luca D. Lung Ultrasonography Score to Evaluate Oxygenation and Surfactant Need in Neonates Treated with Continuous Positive Airway Pressure. JAMA PEDIATR 2015;169(8): e151797.

16. Chiumello D, Mongodi S, Algieri I, Vergani GL, Orlando A, Via G, Crimella F, Cressoni M, Mojoli F. Assessment of Lung Aeration and Recruitment by CT Scan and Ultrasound in Acute Respiratory Distress Syndrome Patients. CRIT CARE MED 2018;46(11):1761-1768. 

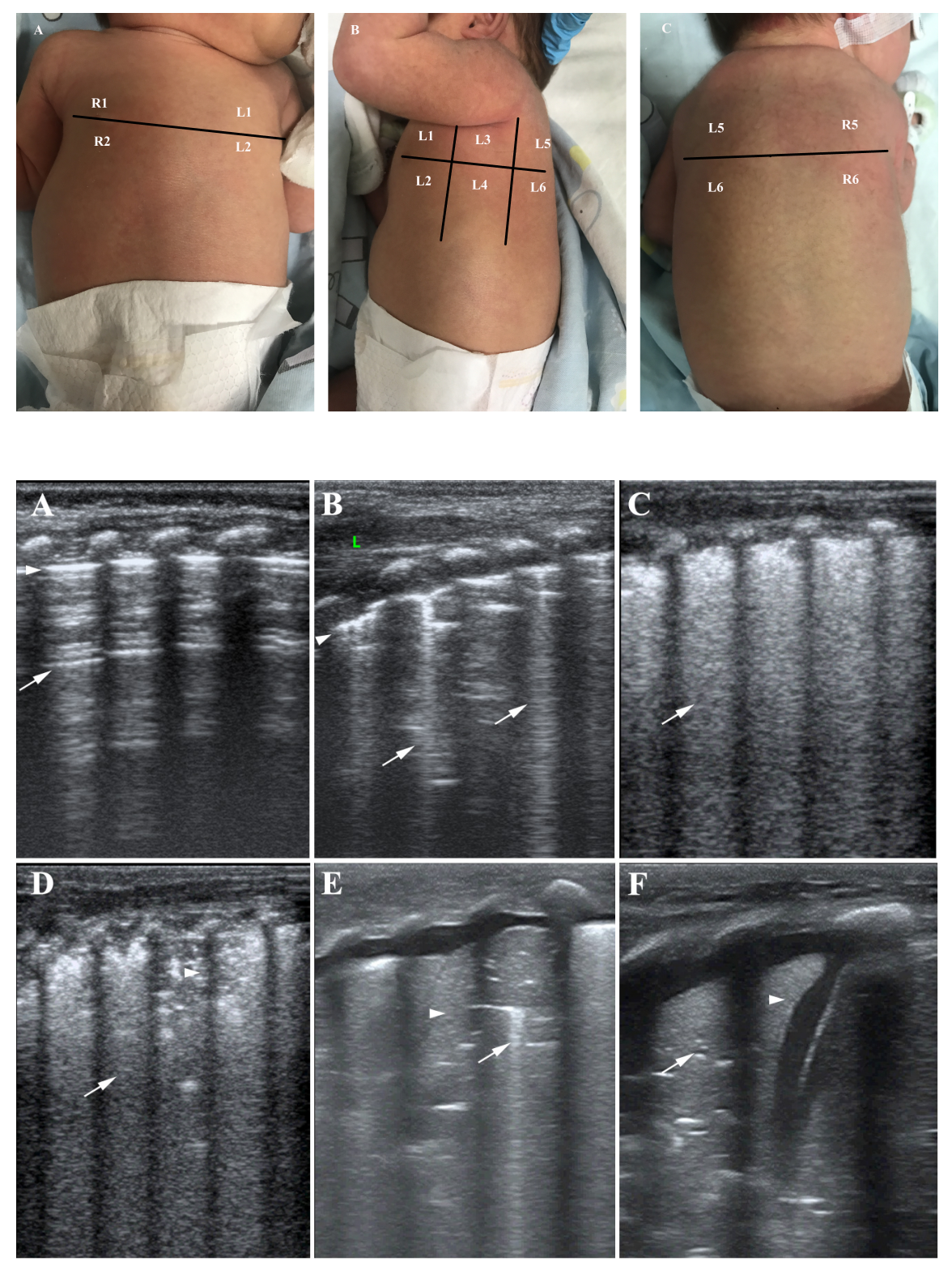

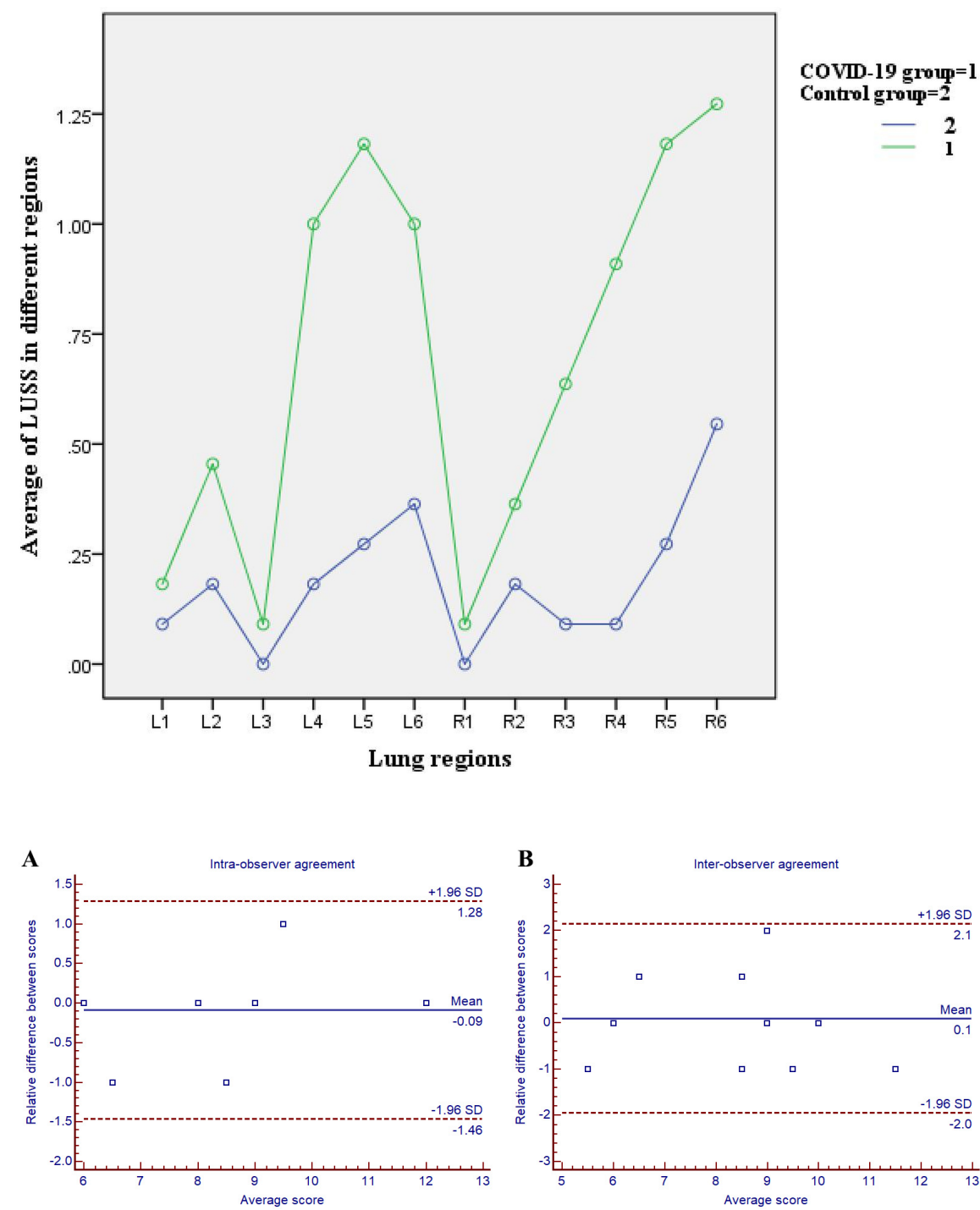

\section{Hosted file}

Table (tab 1-4).pdf available at https://authorea.com/users/361331/articles/482754-bedsidelung-ultrasound-score-luss-on-assessing-pneumonia-in-covid-19-neonates 